\title{
Aetiology of Fever of Unknown Origin: One-Year Experience in a Tertiary Care Hospital of Bangladesh
}

\author{
Rahim MA ${ }^{\mathrm{a}}$, Ahmed AKMS ${ }^{\mathrm{b}}$, Ghosh $\mathrm{SK}^{\mathrm{c}}$, Samad T ${ }^{\mathrm{d}}$, Ahmed JU', Nazneen $\mathrm{S}^{\mathrm{c}}$, Haque HF${ }^{\mathrm{f}}$, \\ Afroze $\mathrm{SR}^{\mathrm{f}}$, Afroz F ${ }^{\mathrm{f}}$, Hossain $\mathrm{MD}^{\mathrm{b}}$, Rahman $\mathrm{MR}^{\mathrm{b}}$, Musa AKM ${ }^{\mathrm{g}}$, Uddin $\mathrm{KN}^{\mathrm{g}}$
}

\begin{abstract}
Background: Fever of unknown origin (FUO) is not an uncommon problem in general medical practice. Sometimes extensive investigations fail to reach an aetiological diagnosis; on the other hand, in few cases, fever resolves spontaneously. This study was aimed to evaluate the aetiology of FUO in a tertiary care setting.

Methods: This cross-sectional study was done in the Department of Internal Medicine of BIRDEM General Hospital, Dhaka, Bangladesh from July 2012 to June 2013.

Results: Among the 33 patients studied (1.23\% of total admissions), 22 (66.7\%) were male. Mean age of the study population was $40.2 \pm 7.9$ years. Most patients (84.8\%) were diabetic. Infection (20, 60.6\%) was the commonest cause, followed by malignancy (9, 27.3\%). Among the infective causes (20), extra-pulmonary tuberculosis (5, 25\%) was the commonest, followed by liver abscess (4, 20\%). Other less common causes were Kala-azar (1), malaria (2), histoplasmosis (2), melioidosis (1), cholecystitis (1), renal abscess (1), rickettsial fever (1), apical dental abscess (1) and infective endocarditis (1). Non-Hodgkin's lymphoma (6), renal cell carcinoma (2) and hepatocellular carcinoma (1) constituted the malignant causes of FUO in this series. Systemic lupus erythematosus was the aetiology of FUO in 1 case. One case remained undiagnosed and 2 patients left hospital before a definite diagnosis could be made.

Conclusion: Extra-pulmonary tuberculosis and non-Hodgkin's lymphoma were the two most common causes of FUO in this study. Repeated history taking, clinical examinations and careful stepwise investigations can diagnose the aetiolgy in most cases of FUO.
\end{abstract}

Key words: aetiology; Bangladesh; fever of unknown origin; pyrexia of unknown origin.

Birdem Med J 2016; 6(1): 7-11

Author Informations

a. Dr. Muhammad Abdur Rahim, FCPS (Medicine), Assistant Professor, Nephrology, BIRDEM.

b. Dr. AKM Shaheen Ahmed, FCPS (Medicine), Dr. Md. Delwar Hossain, MD (Chest), Dr. Md. Raziur Rahman, MD (Medicine), Associate Professor, Internal Medicine, BIRDEM.

c. Dr. Swapan Kumar Ghosh, MBBS, Dr. Surayia Nazneen, FCPS (Medicine), EMO, BIRDEM.

d. Dr. Tabassum Samad, FCPS (Medicine), Junior Consultant, Nephrology, BIRDEM.

e. Dr. Jamal Uddin Ahmed, FCPS (Medicine), Assistant Professor, Internal Medicine, BIRDEM.

f. Dr. Hasna Fahmima Haque, FCPS (Medicine), Dr. Samira Rahat Afroze, FCPS (Medicine), Dr. Farhana Afroz, FCPS (Medicine), Registrar, Internal Medicine, BIRDEM.

g. Prof. AKM Musa, FCPS (Medicine), Prof. Khwaja Nazim Uddin, FCPS (Medicine), Professor, Internal Medicine, BIRDEM.

Address of correspondence: Dr. Muhammad Abdur Rahim, FCPS (Medicine), Assistant Professor, Nephrology, BIRDEM. Email: muradrahim23@yahoo.com

Received: May 22, 2014

Accepted: May 31, 2015

\section{Introduction}

Petersdorf and Beeson in 1961 defined fever of unknown origin (FUO) as "a temperature of $38.3^{\circ} \mathrm{C}\left(101^{\circ} \mathrm{F}\right)$ or greater on several occasions, more than 3 weeks of illness and failure to reach a diagnosis despite 1 week of inpatient investigations". ${ }^{1}$ Durack and Street revised the definition in 1991 to include "3 out-patient visits or 3 days of inpatient investigations" instead of 1 week of inpatient investigation as was in previous criteria ${ }^{1}$ and classified it as being "classical, nosocomial, neutropenic and human immunodeficiency virus (HIV) associated". 2 The causes of pyrexia/fever of unknown origin (PUO/ FUO) exceed 200, but most causes fall under groups of infection, malignancy and collagen vascular disease. . $^{3,4}$ A good number of cases remain undiagnosed ${ }^{4}$ and in few cases fever disappears spontaneously. History and physical examinations may give clues for working diagnostic plan. Along with usual causes, emerging 5,6 and imported ${ }^{7}$ diseases should always be considered 
as differentials. Developments in diagnostic facilities including radiographic and microbiologic methods have changed the spectrum of diseases causing FUO ${ }^{8}$ Still FUO remains the diagnostician's challenge. ${ }^{9}$ In this study we have tried to evaluate the aetiology of FUO in our setting.

\section{Methods}

This cross-sectional study was done in the Department of Internal Medicine of Bangladesh Institute of Research and Rehabilitation in Diabetes, Endocrine and Metabolic Disorders (BIRDEM) General Hospital, Dhaka, Bangladesh from July 2012 to June 2013. Hospitalized adult male patients and non-pregnant female patients aged 18 years and above with prolonged fever ( $>3$ weeks) were initially entitled for the study purpose. Among them who later fulfilled criteria for $\mathrm{FUO}^{2}$ were finally included in this study with the aim to describe the aetiology. Patients with fever for less than three weeks duration and febrile patients with a definite diagnosis during admission were excluded from the study.

After hospitalization detailed history was taken from every patient including area of residence, travel history, history of drug abuse, occupational history, sexual history, history of any surgery, duration of fever, focal features, investigations performed and treatment received before hospitalization. Repeated history was taken by a different medical officer/resident every 2 days. Thorough clinical examination was done and repeated for any new finding development and like history taking examination was repeated by a different medical officer/ resident whenever felt. Reviews of clinical data, performed investigations, given treatments and progress of the patients' condition were done by medical officer/residents supervised by consultant(s) and a new investigation plan was formulated keeping in mind the localizing features, if present and the possible differentials.

Common investigations performed for every patients included complete blood counts (CBC), erythrocyte sedimentation rate (ESR), C-reactive protein (CRP), blood film for malarial parasite, urine routine microscopic examination, chest x-ray postero-anterior view, abdominal ultrasonography (USG), urine and blood cultures and triple antigens. These investigations were done at the earliest time since hospitalization from the standard laboratory services and radiology department of the institute. Other investigations like anti-nuclear antibody (ANA), anti-dsDNA, computed tomographic (CT) scans and other imaging and biopsy were done when necessary as guided by clinical clues and initial investigation reports. For any invasive procedure, informed written consent was taken from the patient according to the existing hospital guidelines and in standard proforma of the institute. Pleural biopsy was done in our department, but image guided biopsies and arthroscopic biopsies required assistance from departments of histopathology, orthopaedics and radiology. Bone marrow biopsies were done by the department of haematology. All these investigations were performed as part of management of the patient.

All data were recorded in preformed semi-structured case record form. Duration from hospitalization to definite diagnosis and total hospital stay were also recorded. Diagnoses were grouped as infection, malignancy, connective tissue disease, miscellaneous and undiagnosed. A case was labeled undiagnosed when all planned investigations were done within a reasonable time of hospital stay and still aetiology could not be identified. Cases that did not fall under infection, malignancy, connective tissue diseases and undiagnosed constituted miscellaneous group. Data were analyzed by using statistical package for social sciences (SPSS) version 15.0. Results were presented in tables.

\section{Results}

During the one-year study period, a total of $33(1.23 \%$ of total admissions) patients fulfilled criteria for FUO. Mean age of the study population was 40.2 \pm 7.9 (range 18-79) years. Males were 22 and females were 11. Most patients were diabetic $(28,84.8 \%)$. Mean duration of fever during hospitalization was 34 days (range 24 days to 7 months). Mean hospital stay and mean time from hospitalization to definite diagnosis were 13 days and 8.2 days respectively.

Besides fever, weight loss, anorexia, cough, headache and abdominal pain were among the most common symptoms. Other features are presented in table I. 
Table I. Presenting features of FUO among the study subjects $(\mathrm{N}=33)$

\begin{tabular}{lcc} 
Symptoms* & Frequency & Percentage \\
\hline Weight loss & 26 & 78.8 \\
Anorexia & 21 & 63.6 \\
Cough & 17 & 51.5 \\
Headache & 9 & 27.3 \\
Abdominal pain & 12 & 36.4 \\
Chest pain & 7 & 21.2 \\
Sputum production & 5 & 15.2 \\
Palpitation & 2 & 6.1 \\
Joint pain & 2 & 6.1 \\
Dysuria & 4 & 12.1 \\
Rash & 1 & 3 \\
\hline
\end{tabular}

*All patients had more than one symptom
Among the study subjects, $32(97 \%)$ had anaemia and $16(48.5 \%)$ had neutrophilic leukocytosis. ESR was high $\left(>20 \mathrm{~mm}\right.$ in $1^{\text {st }}$ hour) in all cases and it was $>100 \mathrm{~mm}$ in 1 st hour in $7(21.2 \%)$ cases. CRP was raised in $26(78.8 \%)$ cases. Malarial parasite was found in blood film in one case. Urine routine examination showed proteinuria, glycosuria, pyuria and haematuria in $24(72.7 \%), 5$ $(15.2 \%), 5(15.2 \%)$ and $4(12.1 \%)$ cases respectively. Chest x-ray and abdominal USG were abnormal in 5 $(15.2 \%)$ and $12(36.4 \%)$ cases respectively. CT scan was done in 19 cases and found abnormal (CT abdomen in 16 cases and $\mathrm{CT}$ chest in 3 cases).

Serological tests confirmed diagnoses in 4 cases and tissue diagnosis (including biopsy and histopathology and fine needle aspiration cytology) in 13 cases (table II). In 1 case acid fast bacilli (AFB) was found in urine test and Mycobacterium tuberculosis polymerase chain reaction (MTB-PCR) was positive in another 1 case.

Infection $(20 / 33,60.6 \%)$ and malignancy $(9 / 33,27.3 \%)$ were the two most common causes of FUO in this study. Non-Hodgkin's lymphoma and extra-pulmonary tuberculosis comprised one-third of all cases. Other causes are shown in table II.

Table II. Causes of FUO and confirmatory tests of the study subjects ( $\mathrm{N}=33$ )

\begin{tabular}{llcc} 
Aetiology & Confirmatory test(s) & Frequency & Percentage \\
\hline Infection & & 20 & 60.6 \\
Tuberculosis & & 5 & 15.2 \\
$\quad$ Pleura & Pleural biopsy & 1 & 3 \\
$\quad$ Genito-urinary & Urine AFB, MTB-PCR & 2 & 6.1 \\
$\quad$ Aritonitis & Laparoscopic biopsy & 1 & 3 \\
Liver abscess & Arthroscopic biopsy & 1 & 3 \\
Calculus cholecystitis & USG, CT scan, USG guides aspiration & 4 & 12.1 \\
Renal abscess & USG & 1 & 3 \\
Kala-azar & CT scan & 1 & 3 \\
Malaria & Bone marrow study & 1 & 3 \\
Histoplasmosis & Blood film, ICT for malaria & 2 & 6.1 \\
Melioidosis & Bone marrow, CT guided FNAC from suprarenal gland & 2 & 6.1 \\
Rickettsial fever & Pus culture, synovial fluid culture & 1 & 3 \\
Apical dental abscess & Triple antigen & 1 & 3 \\
Infective endocarditis & Drainage of pus & 1 & 3 \\
Malignancy & Blood culture, echocardiography & 1 & 3 \\
Non-Hodgkin's lymphoma & Lymphnode biopsy, bone marrow trephine biopsy & 6 & 27.3 \\
Hepatocellular carcinoma & CT guided FNAC, alpha-fetoprotein & 18.2 \\
Renal cell carcinoma & USG, CT scan & 1 & 3 \\
Connective tissue disease & & 2 & 6.1 \\
Systemic lupus erythematosus & ANA, anti-dsDNA & 1 & 3 \\
Miscellaneous* & & 1 & 3 \\
Undiagnosed & & 2 & 6.1 \\
\hline
\end{tabular}

*two patients were discharged against medical advice before a definite diagnosis could be made 
Table III. Comparison of aetiology of FUO in eight different series

Author, year, (ref) Total number Infection Malignancy Connective Miscellaneous Undiagnosed

\begin{tabular}{lcccccc} 
& of patients & $(\%)$ & $(\%)$ & tissue disease (\%) & $(\%)$ & $(\%)$ \\
\hline Petersdorf \& Beeson, 1961,(1) & 100 & 36 & 19 & 15 & 23 & 7 \\
*Haq SA et al, 1996, (20) & 212 & 64 & 12 & 11 & 6 & 7 \\
Kejariwal D et al, 2001, (22) & 100 & 53 & 17 & 11 & 5 & 14 \\
Liu KS etal, 2003, (5) & 78 & 42.3 & 6.4 & 20.5 & 7.7 & 23.1 \\
Alavi SM et al, 2009,(12) & 84 & 48.9 & 8.3 & 17.8 & 7 & 16.7 \\
Khattak MI et al, 2011,(21) & 100 & 57 & 10 & 12 & 4 & 17 \\
Bandyapadhyay D et al, 2011,(13) & 164 & 58.53 & 22 & 11 & - & $8.5^{* *}$ \\
Rahim MA et al, 2016, (current) & 33 & 60.6 & 27.3 & 3 & 6.1 & 3 \\
\hline
\end{tabular}

*Prolonged fever ( $>3$ weeks), **up to the end of one month

\section{Discussion}

Aetiology of FUO is not exhaustive and it largely depends upon geographic area, age group, economic status and over times. ${ }^{10-12}$ Infection, malignancy and connective tissue diseases constitute the majority of causes. Over times incidence of neoplastic diseases are increasing ${ }^{13}$ and infection is decreasing, possibly because of improved diagnostic tools and improvement of health care facilities resulting in less incidence of infections. Sporadic cases of FUO have been described from Bangladesh having diverse aetiology including Behcet's disease ${ }^{14}$, Castleman's disease ${ }^{15}$, renal cell carcinoma $^{16}$, histoplasmosis ${ }^{17}$, melioidosis ${ }^{18}$, leishmaniasis ${ }^{19}$ etc. Haq SA et al. in 1996 described 212 cases of prolonged pyrexia ( $>3$ weeks) from Dhaka, Bangladesh, of whom 159 fulfilled criteria for FUO, and $87 \%$ of all had infection, malignancy and connective tissue disease as aetiology. ${ }^{20}$

Mean age of our study population was just over 40 years with males being twice more common than females. Mean age of study population in different series from Bangladesh $^{20}$, Iran ${ }^{12}$, Pakistan ${ }^{21}$ and Taiwan ${ }^{5}$ were 33.8, 49.2, 40 and 49 years respectively with male predominance in every series except Pakistan. Mean duration of fever before admission, mean hospital stay and mean time since hospitalization to a definite diagnosis were 34, 13 and 8.2 days respectively in current study. Mean hospital stay and time needed to reach a diagnosis were 14.47 and 5.64 days in one study in eastern India. ${ }^{13}$
Microbiological tests, serology and immunology, radiological tests, biopsy and histopathology confirmed diagnosis in 4, 4, 9 and 13 cases respectively in our study. One (3\%) case remained undiagnosed despite appropriate investigations, which was much less than other series. $1,5,12,20,21$

Infection was the commonest cause of FUO in our study and tuberculosis comprised one-fourth of infective cases. Tuberculosis was the commonest cause in most other series. 1,5,12,20-22 Comparison of aetiology of 8 different series, mostly from Asian countries is shown in table III. It is to be mentioned that overall incidence of infection is reduced, malignancy has risen and number of undiagnosed cases are also reduced. Uncommon and rare diseases are coming as aetiology because of improved diagnostic facilities. ${ }^{13,17,18}$

\section{Limitations}

This study had few limitations. Number of cases was small and most patients were diabetic. Whether aetiology of FUO is similar among non-diabetic population is not clear. A large, multi-center study including large number of patients will give better representative picture from Bangladesh.

\section{Conclusions}

In conclusion, it can be said that by using proper clinical data, routine laboratory tests and careful, focused and sometimes invasive tests can identify aetiology in most cases of FUO. Infection specially extra-pulmonary tuberculosis, still remains as one of the commonest causes of FUO and incidence of malignancy is 
increasing. Every effort should be given to identify the aetiology in FUO, so that specific treatment can be offered.

\section{Acknowledgement}

We express our acknowledgement to everybody involved in managing the patients with FUO during hospital stay and collecting data for the study purpose.

\section{Conflict of Interest: None.}

\section{References}

1. Petersdorf RG, Beeson PB. Fever of unexplained origin: report on 100 cases. Medicine 1961;40:1-30.

2. Durack DT, Street AC. Fever of unknown origin: reexamined and redefined. In: Remington JS, Swartz MN, eds. Current Clinical Topics in Infectious Diseases. Boston, Mass: Blackwell Scientific Publications Inc; 1991;11: 35-51.

3. Jha A, Sarda R. Value of bone marrow examination in pyrexia of unknown origin. J Path Nepal 2013;3:447-51.

4. Amow PM, Flaherty JP. Fever of unknown origin. Lancet 1997;350:575-80.

5. Liu KS, Sheng WH, Chen YC, Chang SC, Hsieh WC. Fever of unknown origin: a retrospective study of 78 adult patients in Taiwan. J Microbiol Immunol Infect $2003 ; 36: 243-47$

6. Gogia A, Dugga L, Dutta S. An unusual aetiology of PUO. J Assoc Phy India 2011;59:47-49.

7. Hoseini MJ, Jafari NJ, Poornaki AM, Sadraei J, Rezavand B, Hamedanchi A et al. Fever of unknown origin with final diagnosis of imported Malaria - A case study. Int J Travel Med Global Health 2013;1(2):39-41.

8. Tabak F, Mert A, Celik AD, Ozaras R, Altiparmak MR, Ozturk R et al. Fever of unknown origin in Turkey. Infection 2003;31(6):417-20.

9. Petersdorf RG. Fever of Unknown Origin. An Old Friend Revisited [Editorial]. Arch Intern Med 1992;152:21-22.

10. Ahmed JU, Rahim MA, Hossain MD, Ahmed AKMS, Musa AKM, Uddin KN. Focused Diagnostic Approach to Patient with Fever of Unknown Origin (FUO): A Review. Birdem Med J 2013; 3(1): 27-34.

11. Joshi N, Rajeshwari K, Dubey AP, Singh T, Kaur R. Clinical spectrum of fever of unknown origin among Indian children. Ann Trop Paediatr 2008;28:261-66.
12. Alavi SM, Nadimi M, Sefidgaran G, Papi MH, Zamani GA. Clinical spectrum and diagnostic tools of fever of unknown origin among hospitalized patients in Razi Hospital (2006-2008), Ahvaz. Jundishapur J Microbiol 2009;2(4):152-57.

13. Bandyapadhyay D, Bandyapadhyay R, Paul R, Roy R. Aetiological study of fever of unknown origin in patients admitted to medicine ward of a teaching hospital of eastern India. J Glob Infect Dis 2011;3(4):329-33.

14. Samad T, Ahmed JU, Rahim MA, Musa AKM, Uddin KN, Ahmed AKMS et al. Behcet's Disease Presenting as Fever of Unknown Origin: A Case Report. Birdem Med J 2013; 3(1):50-53.

15. Sultana N, Chowdhury RK, Chowdhury NA, Fariduddin M, Jamal N, Hasanat MA. Castleman's Disease - A Rare Cause of Pyrexia of Unknown Origin (PUO): Case Report. BSMMU J 2009; 2(2): 85-87.

16. Ahmed JU, Afroze SR. Medical Quiz: Images. Birdem Med J 2014;4(1):56-58.

17. Yasmin R, Rahim MA, Haque HF, Dewan P, Ahmed JU, Ahmed AKMS et al. Disseminated Histoplasmosis as Presenting Feature of Acquired Immunodeficiency Syndrome: A Case Report. Bangladesh J Medicine 2013; 24(2): 78-81.

18. Rahim MA, Afroze SR, Barai L, Uddin KN. Melioidosis in Bangladesh - Truly Uncommon or Uncommonly Diagnosed? A Case Report. Birdem Med J 2015;5(suppl 1):49-51

19. Salma U, Khan AH, Rahaman MFU, Irshadullah M, Shumy F, Chowdhury MAJ. Leishmanial Hepatitis with Chronic Hepatitis B Infection Treated Successfully with Liquid Form of Liposomal Amphotericin B: A Case Report. BSMMU J 2012;5(1):55-56.

20. Haq SA, Alam MN, Hossain SM, Dhar UK, Rahim S, Rahman $\mathrm{M}$ et al. A study of prolonged pyrexia in Dhaka. Bangladesh Med Res Counc Bull 1996; 22(1): 33-42.

21. Khattak MI, Ishaq T, Amin S, Rehman SU, Shabbir G. Pyrexia of unknown origin: aetiologic frequency in a tertiary care hospital. Gomal J Med Sci. 2011;9(1):11114.

22. Kejariwal D, Sarkar N, Chakraborti SK, Agarwal V, Roy S. Pyrexia of unknown origin: A prospective study of 100 cases. J Postgrad Med 2001; 47: 104-107. 